\title{
USE OF SATURATION-RELAY FEEDBACK FOR AUTOTUNE IDENTIFICATION
}

\author{
SHIH-HAUR SHEN, HONG-DEN YU and CHENG-CHING YU* \\ Department of Chemical Engineering, National Taiwan Institute of Technology, Taipei, Taiwan 10672, \\ R.O.C.
}

(First received 5 December 1994; accepted 8 September 1995)

\begin{abstract}
Despite the success of the relay-feedback system in autotune identification, it is well known that an ideal (on-off) relay-based identification can lead to significant error in the ultimate gain and ultimate frequency. Instead of taking remedial action after relay-feedback experiments, an approach is taken to re-design the experiment such that a better estimate of $K_{u}$ and $\omega_{u}$ can be achieved throughout the experiment. In this work, the saturation relay is employed instead of the conventional ideal relay. The analyses show that the saturation relay gives significant improvement in the identification of $K_{u}$ and $\omega_{u}$. However, too small a slope in the saturation-relay may also fail to generate sustained oscillation. A procedure is tested on linear and nonlinear systems for system identification and multivariable autotuning. Using the proposed method significant improvement can be achieved while maintaining the simplicity of the relay feedback test.
\end{abstract}

\section{INTRODUCTION}

Autotuning of PID controllers based on relay-feedback tests has received a great deal of attention recently (Luyben, 1987; Hägglund and Åström, 1991). It identifies the important dynamic information, ultimate gain $\left(K_{u}\right)$ and ultimate frequency $\left(\omega_{u}\right)$, in a straightforward manner. The success of this type of autotuners lies on the fact that it is simple and reliable. The appealing feature of the relay-feedback autotuning has lead to a number of commercial autotuners (Hägglund and Åström, 1991) and industrial applications (Papastathopoulou and Luyben, 1990). Extensions of relay-feedback systems to monitoring (Chiang and Yu, 1993) and nonlinear control ( $L$ in and Yu, 1993; Luyben and Eskinat, 1994) has been made. Moreover, the autotuning multiloop PI controllers has also been proposed (Shen and Yu, 1994; Loh and Vasnani, 1994).

Luyben (1987) pioneered the use of relay-feedback tests for system identification. The ultimate gain and ultimate frequency from the relay-feedback test are used to fit a typical transfer function (e.g. first-, second- or third-order plus time-delay system). This identification procedure is called ATV method. It was applied successfully to a highly nonlinear process, e.g. high-purity distillation column. Despite the apparent success of autotune identification, it can lead to significant errors in the ultimate gain and ultimate frequency approximation (e.g. $5-20 \%$ error in $K_{u}$, see Chiang et al. (1992)) for typical transfer functions in process control system. The errors arise because of the use of linear approximation (describing function analysis) to a nonlinear element. The square type of output

* Corresponding author. Tel.: 886-2-737-6620. Fax: 886-2737-6644. E-mail: ccyu@ch.ntit.edu.tw. from the relay is approximated with the principal harmonic form of the Fourier transform (Antherton, 1982; Chiang et al., 1992) and the ultimate gain is estimated accordingly. Therefore, several attempts have been proposed to overcome this inaccuracy. $\mathrm{Li}$ et al. (1991) use two relay tests to improve the estimation of $K_{u}$ and $\omega_{u}$. Chiang et al. (1992) employ the concept of a discrete-time system to give a better estimation of $\omega_{u}$. Notice that, in these attempts, ideal (on-off) relay is employed in these experiments and modifications are made after the experiments are completed. Since the major source of the error comes from sine-wave approximation of a square type of oscillation, a straightforward approach to overcome this inaccuracy is to modify the experiment $i t s e l f$ (not to make remedial action afterward), i.e. to produce a more sine-wave-like output by re-designing the relay.

The purpose of this work is to devise an experimental design in relay-feedback system such that more accurate estimate of ultimate gain and ultimate frequency can be achieved. In this work, saturation relay is employed. This paper is organized as follows. Section 2 describes the theory of saturation-relay feedback system. Section 3 derives the autotune identification procedures. A linear system and a nonlinear distillation column are used to test the effectiveness of the proposed method and the proposed autotune identification is also applied to MIMO autotuning in Section 4 and is followed by the conclusion.

\section{ANALYSIS}

2.1. Conventional approach: ideal (on-off) relay

Autotuning based on relay feedback can be analyzed via block diagram. Consider a feedback system (Fig. 1) where $G(s)$ is a linear transfer function and $N$ is a nonlinear element. If the input signal $(e(t))$ to 


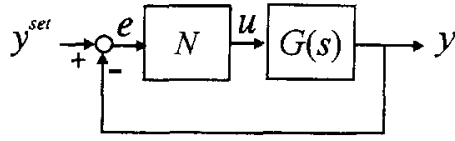

Fig. 1. Nonlinear feedback system.

the nonlinear element is a sinusoidal wave:

$$
e(t)=a \sin \omega t
$$

where $a$ is the magnitude of sinusoidal wave, the output signal $(u(t))$ of the nonlinear element is a square wave (Fig. 2). Since most control system analyses are based on linear theory, Fourier transformation is useful in this regard. The output of the nonlinear element can be expressed as

$$
u(t)=A_{0}+\sum_{n=1}^{\infty} A_{n} \cos n \omega t+B_{n} \sin n \omega t
$$

where

$$
\begin{aligned}
& A_{0}=\frac{1}{2 \pi} \int_{0}^{2 \pi} u(t) \mathrm{d}(\omega t) \\
& A_{n}=\frac{1}{\pi} \int_{0}^{2 \pi} u(t) \cos n \omega t \mathrm{~d}(\omega t) \\
& B_{n}=\frac{1}{\pi} \int_{0}^{2 \pi} u(t) \sin n \omega t \mathrm{~d}(\omega t) .
\end{aligned}
$$

Because the output, $u(t)$, is an odd-symmetric function (i.e. $N(a)$ is unbiased and symmetric to the origin), the coefficients $A_{0}$ and $A_{n}$ are equal to zero (i.e. $A_{0}=0$ and $A_{n}=0, \forall n$ ). Therefore, eq. (2) becomes

$$
u(t)=\sum_{n=1}^{\infty} B_{n} \sin n \omega t .
$$

Furthermore, if an ideal relay is employed (Fig. 2), then the coefficients $B_{n}$ become

$$
B_{n}= \begin{cases}\frac{1}{n} \frac{4 h}{\pi}, & n=1,3,5, \ldots \\ 0, & n=2,4,6, \ldots\end{cases}
$$

The describing function analysis provides a tool for frequency domain analysis for this nonlinear system. Only the principal harmonic is employed for the linear equivalence, i.e. only the first Fourier coefficient is used for frequency domain analysis. Therefore, the describing function is

$$
N(a)=\left(B_{1}+j A_{1}\right) / a .
$$

For the ideal relay, since $A_{1}=0$ and $B_{1}=4 h / \pi$, we have

$$
N(a)=4 h / \pi a .
$$

Since sustained oscillation is generated from a relayfeedback test (e.g. Fig. 2), this frequency corresponds to the limit of stability, i.e.

$$
1+G\left(j \omega_{u}\right) N(a)=0
$$

(A) Ideal Relay

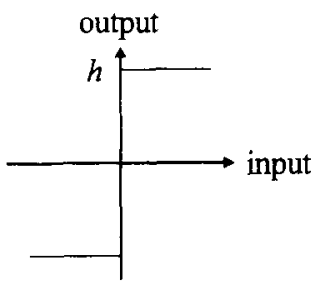

(B) Input-Output responses

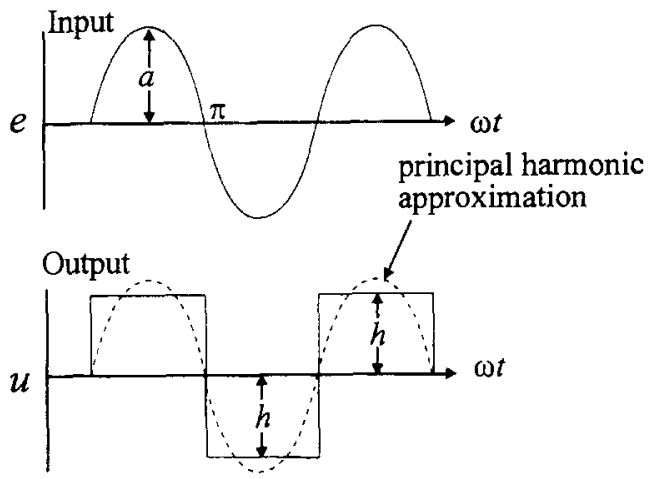

Fig. 2. Input-output relationship for ideal (on-off) relay.

or the ultimate gain $\left(K_{u}\right)$ becomes

$$
\begin{aligned}
K_{u} & =-1 / G\left(j \omega_{u}\right) \\
& =N(a) \\
& =4 h / \pi a .
\end{aligned}
$$

Part of the success of the autotune identification comes from the fact that $K_{u}$ and $\omega_{u}$ can be read directly from the experimental results [e.g. Fig. 2(B)].

The results of eq. (11) clearly indicate that the ultimate gain $\left(K_{u}\right)$ is estimated from the amplitude ratio of two sinusoidal waves at a given frequency $\left(\omega_{u}\right)$ (i.e. $u(t)=(4 h / \pi a) \sin \omega t$ over $e(t)=a \sin \omega t)$. Obviously, the output of the relay is a square wave instead of a sinusoidal wave. This leads to erroneous result in the estimated ultimate gain. Figure 2 shows the corresponding input-output relationship for an ideal relay. Here, the principal harmonic is used to approximate a square wave [Fig. 2(B)]. Chiang et al. (1992) point out that the truncation of the higher-order terms (i.e. $n=3,5,7, \ldots$ ) affects the ultimate gain and ultimate frequency estimation. Mathematically, it is difficult to include the high-order terms in a linear analysis. Instead of including higher-order terms, a straightforward approach is to redesign the relayfeedback experiment. In other words, it is helpful to devise an experiment such that the output response of the relay is more sine-wave-like (less square-wavelike).

\subsection{Alternative: saturation-relay feedback}

Since the square-wave output [e.g. Fig. 2(B)] comes from the abrupt change at the zero point [i.e. 
$e(t)=0$ in Fig. 2] of an ideal relay, the saturation relay provides an attractive alternative for a smooth transition around the zero point as shown in Fig. 3(A). The saturation relay is characterized by two parameters: relay height $(h)$ and a slope $(k)$ [Fig. 3(A)]. Therefore, the input of the relay is limited by $\bar{a}$ where

$$
\bar{a}=h / k
$$

i.e. if the input to the relay is less than $\bar{a}(|e| \leqslant \bar{a})$, then the output is proportional to the input with a factor $k$ :

$$
u=k \cdot e .
$$

However, if the input to the relay is greater than $\bar{a}(|e|>\bar{a})$, then the output of the relay is limited by $h$

$$
u=h
$$

or

$$
u=-h
$$

With the saturation relay inserted in the feedback loop, the output of the relay is less square-wave-like response for a sine-wave, with an upper (or a lower) limit and the height of the output response is limited by $h(h=k \bar{a})$.

The output of the saturation relay can be characterized analytically. Consider a saturation-relay feedback system to the nonlinear element to be a sinusoidal wave with an amplitude $a$ [Fig. 3(B)], i.e.

$$
e(t)=a \sin \omega t
$$

The output to the nonlinear element, $u(t)$, looks like a truncated sinusoidal wave and the closeness of this output response to a sine wave depends a great deal on the slope $k$ chosen. The angle $\gamma$ (Fig. 4) gives a simple measure to characterize the relay output.

$$
\gamma=\sin ^{-1}(\bar{a} / a)
$$

Since the relay output is a periodic function, considering half period, if the phases lie between $\gamma$ and $\pi-\gamma$, the output is equal to $h$ and the sine-wave-like responses remain, for $\omega t<\gamma$ and $\omega t>\pi-\gamma$, as shown in Fig. 4. Obviously, the $\gamma$ values depend on the slope $k$. If $k \rightarrow \infty$, we have

$$
\gamma=\lim _{k \rightarrow \infty} \sin ^{-1}((h / k) / a)=0 .
$$

Then, the output becomes a square wave. With this measure, the relay output can be expressed as

$$
\begin{aligned}
& u(t)= \\
& \begin{cases}\frac{h}{\sin \gamma} \sin \omega t & 0 \leqslant \omega t<\gamma \text { and } \pi-\gamma<\omega t \leqslant \pi \\
h & \gamma \leqslant \omega t \leqslant \pi-\gamma .\end{cases}
\end{aligned}
$$

(A) Saturation Relay

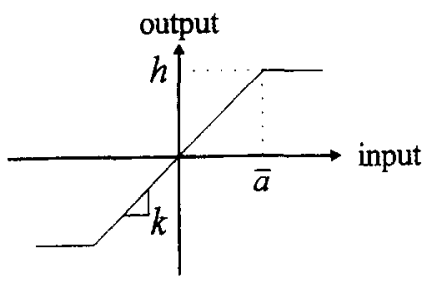

(B) Input-Output responses

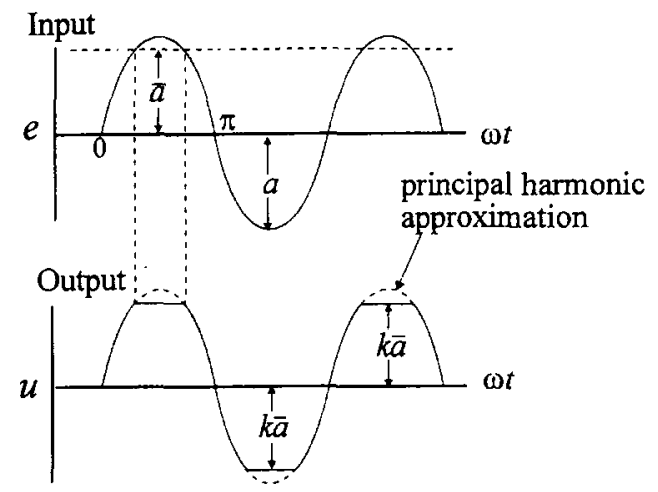

Fig. 3. Input-output relationship for saturation relay.

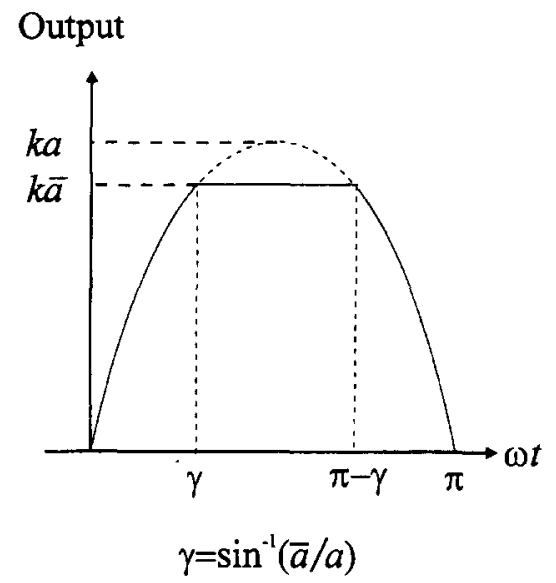

Fig. 4. Graphical interpretation of the angle $\gamma$.

Since the principal harmonic is employed for linear approximation, the Fourier transformation of $u(t)$ is useful for the purpose analysis:

$$
u(t)=\sum_{n=1}^{\infty} B_{n} \sin n \omega t
$$

where

$$
B_{n}=\frac{2}{\pi} \int_{0}^{\pi} u(t) \sin n \omega t \mathrm{~d}(\omega t)
$$

Since the term $\gamma$ plays an important role in the frequency domain analysis, the relationship between 
$\gamma$ and frequency responses is studied. Consider the following cases:

(A) $0<\gamma<\pi / 2(\infty>k>h / a)$. For this general case, substituting $u(t)$ [eq. (19)] into eq. (21), we have et al., 1992) and the even coefficients remain zero. Thus, the describing function becomes

$$
N(a)=\frac{2 h}{\pi}\left(\frac{1}{\bar{a}} \sin ^{-1}\left(\frac{\bar{a}}{a}\right)+\frac{\sqrt{a^{2}-\bar{a}^{2}}}{a^{2}}\right) .
$$

$B_{n}= \begin{cases}\frac{2 h}{\pi}\left[\frac{1}{\sin \gamma}\left(\frac{\sin (1-n) \gamma}{1-n}-\frac{\sin (1+n) \gamma}{1+n}\right)-\frac{1}{n}(\cos n(\pi-\gamma)-\cos \gamma)\right], & n=1,3,5, \ldots \\ 0, & n=2,4,6, \ldots\end{cases}$

The expression for the odd coefficients (e.g. $B_{1}$, $B_{3}, B_{5}, \ldots$ ) differs from that of an ideal relay (Chiang
Since the higher-order terms [eq. (22)] are neglected, Fig. 5 clearly shows that the principal harmonic
(A) $\gamma=0 \quad(\bar{a}=0 \cdot a)$

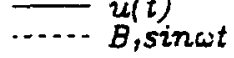

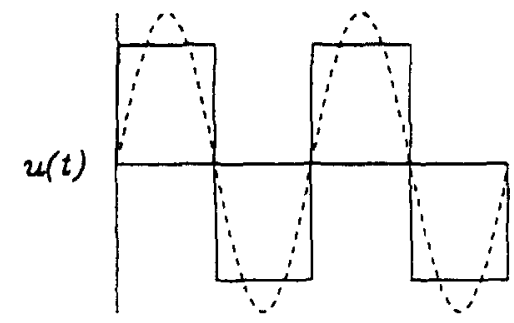

(B) $\gamma=\pi / 4 \quad\left(\bar{a}=2^{1 / 2} / 2 \cdot a\right)$

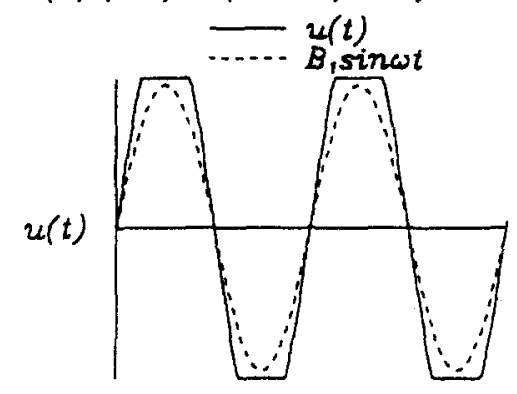

(C) $\gamma=\pi / 2(\bar{a}=1 \cdot a)$

$$
-\ldots, t(t)
$$

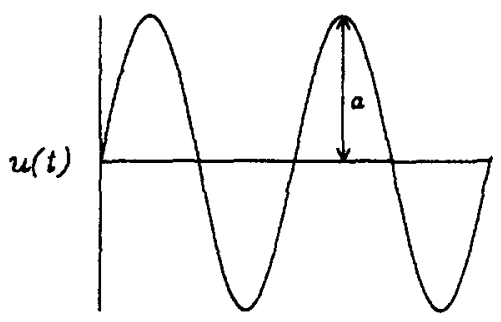

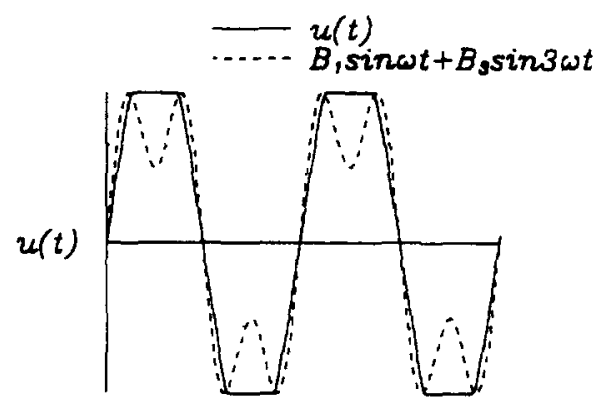

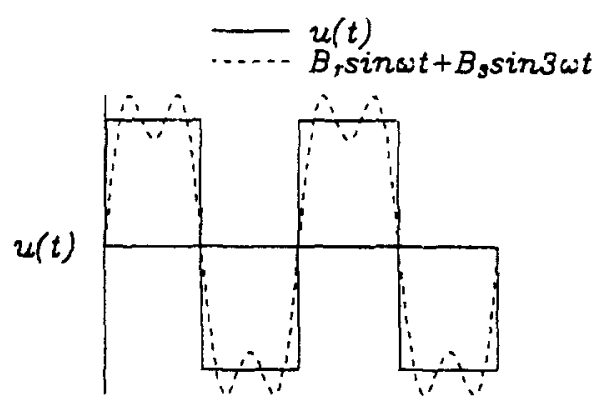

$u(t)$

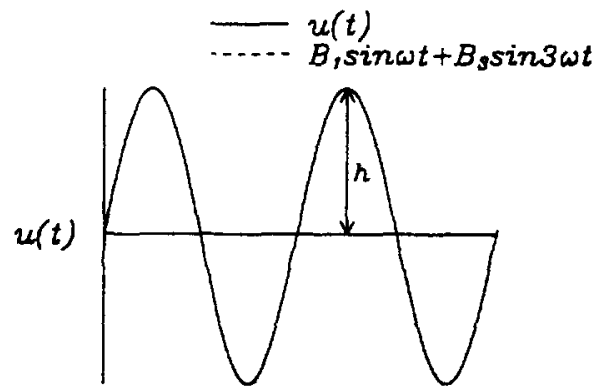

Fig. 5. The wave shape of output to the nonlinear element for different slope. 
approximation cannot exactly describe the output response [e.g. Fig. 5(B)].

(B) $\gamma=0(k \rightarrow \infty)$. Let us first consider an asymptotic case when the slope of saturation relay approaches infinity (i.e. $k \rightarrow \infty$ ) [Fig. 3(A)]. In this case, $\bar{a}$ becomes zero [eq. (12)], $\gamma$ becomes zero [eq. (16)], and, subsequently, the saturation relay is reduced to an ideal relay (Fig. 2). The coefficients $\left(B_{n}\right)$ of Fourier expansion can be derived from eq. (22). After some algebraic manipulation, we have

$$
\begin{gathered}
B_{n}=\lim _{\gamma \rightarrow 0} \frac{2 h}{\pi}\left\{\frac{1}{\sin \gamma}\left[\frac{\sin (1-n) \gamma}{1-n}-\frac{\sin (1+n) \gamma}{1+n}\right]\right. \\
\left.\quad-\frac{1}{n}[\cos n(\pi-\gamma)-\cos \gamma]\right\} \\
=\frac{1}{n} \frac{4 h}{\pi}, \quad n=1,3,5, \ldots
\end{gathered}
$$

and

$$
B_{n}=0, \quad n=2,4,6, \ldots
$$

Since the principal harmonic $\left(B_{1}\right)$ is employed for the describing function analysis,

$$
\begin{aligned}
N(a) & =\lim _{\bar{a} \rightarrow 0} \frac{2 h}{\pi}\left(\frac{1}{\bar{a}} \sin ^{-1}\left(\frac{\bar{a}}{a}\right)+\frac{\sqrt{a^{2}-\bar{a}^{2}}}{a^{2}}\right) \\
& =\frac{4 h}{\pi a}
\end{aligned}
$$

Again, the principal harmonic approximation cannot exactly describe the output response [e.g. Fig. 5(A)].

(C) $\gamma=\pi / 2 \quad(k=h / a)$. Let us consider another asymptotic case, i.e. the slope is carefully chosen such that $\bar{a}=a$ (or $k=h / a$ ). In this case, the output of the relay is exactly a sine-wave [e.g. Fig. $5(\mathrm{C})]$. Therefore, the Fourier coefficients can be found by substituting $\gamma=\pi / 2$ into eq. (22). Here, we have

$$
B_{n}= \begin{cases}h & n=1 \\ 0 & \text { otherwise }\end{cases}
$$

For this case, only primary harmonic term exists $\left(B_{1}=h\right.$ and $B_{n}=0$ for $n \geqslant 2$ ), the principal harmonic approximation gives exact solution. Thus, the output of the saturation relay is

$$
u(t)=h \sin \omega t .
$$

Equation (28) shows that, the saturation relay gives a pure sinusoidal wave and output lags behind the input by $-180^{\circ}$. Obviously, this is exactly the conventional sine-wave test and from the definition, the ultimate gain

$$
K_{u}=h / a \text {. }
$$

From the describing function analysis, $N(a)$ can be found by substituting $\gamma=\pi / 2$ into eq. (23):

$$
N(a)=h / a \text {. }
$$

Comparing eq. (30) with eq. (29), it is clear that no approximation is involved in this estimation.

The analyses of these three cases show that the saturation relay is a generalization of the ideal relay. More importantly, better estimates of $K_{u}$ and $\omega_{u}$ can be achieved by adjusting the slope of the relay. For example, when $\gamma=0$, we have an ideal relay and as $\gamma$ increases to $\pi / 2$ the experiment becomes a conventional sine-wave test. Therefore, it provides the flexibility in finding more accurate $K_{u}$ and $\omega_{u}$

\subsection{Potential problem}

The improvement of the estimates of $K_{v}$ and $\omega_{u}$ does not come without any potential problem. One possible case is that if the slope is chosen too small (or $a$ is smaller than $\bar{a}$ ), then a limit cycle may not exist. This can be analyzed from frequency responses. Notice that the condition for the existence of sustained oscillation is

$$
1+G(j \omega) N(a)=0
$$

or

$$
G(j \omega)=-1 / N(a) .
$$

Equation (32) can be solved by plotting and the intersection corresponds to the crossover point $\left(K_{u}\right.$ and $\omega_{\mathrm{w}}$ ). For an ideal relay, the $-1 / N$ loci starts from the origin and goes toward $-\infty$ as $N$ increases (e.g. starting from the point a toward left in Fig. 6). In terms of a saturation relay, the starting point of the $-1 / N$ loci corresponds to $-1 / k$ (Fig. 6). As we decrease the slope, the starting point moves to the left accordingly. If the starting point moves to $b$, we still have an intersection and a limit still exists. However, if the slope is decreased further and the starting point is moved over the point $\mathrm{c}$, then $-1 / N$ does not intersect $G(j \omega)$ any longer and we do not have a limit cycle. Therefore, there exist a critical slope $\left(k_{\min }\right)$ such that when the slope of the saturation relay is smaller than this value, the feedback system cannot generate sustained oscillation. On the other hand, if the slope is chosen too large, the relay approaches an ideal relay and the improvement in the estimate of $K_{u}$ and $\omega_{u}$ disappears. Therefore, a trade-off has to be made in

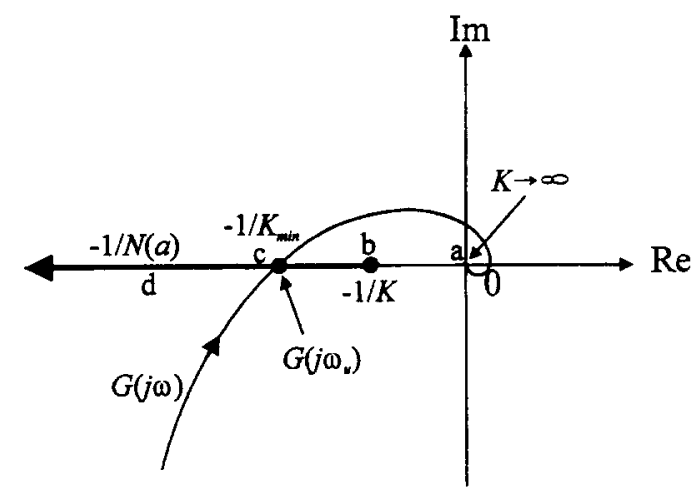

Fig. 6. Loci of $G(j \omega)$ and $-1 / N(a)$ 
the selection of the slope. Furthermore, this critical slope $\left(k_{\min }\right)$ is related to $|G(j \omega)|$ :

$$
k_{\min }=1 /\left|G\left(j \omega_{u}\right)\right| .
$$

The following example illustrates the trade-off.

Example 1: WB column (Wood and Berry, 1973).

$$
G(s)=\frac{x_{D}}{R}=\frac{12.8 e^{-s}}{16.8 s+1} .
$$

This first-order plus dead time system has the following ultimate properties: $K_{u}=2.1$ and $\omega_{u}=1.608$. If an ideal relay is used $(k \rightarrow \infty)$, the test gives $R_{u}=1.71$ and $\hat{\omega}_{u}=1.615$. This test shows an almost $-20 \%$ error in the estimate of $K_{u}$. Furthermore, neither the input $\left(x_{D}\right)$ nor the output $(R)$ of the relay shows sine-wave-like responses [Fig. 7(A)]. If the slope $k$ decreases to five (this gives $\gamma=18$ ), then the system responses behave more sine-wave-like [Fig. 7(B)] and the estimate $\hat{K}_{u}=1.94$ ( $8 \%$ error). Obviously, an improvement in the estimate of $K_{u}$ can be seen using the saturation relay. If the slope decreases further to the critical slope $\left(k=k_{\min }=2.1\right)$, the input and output of the relay look exactly like a sine-wave [Fig. 7(C)] and $\hat{K}_{u}=2.098$ and $\hat{\omega}_{u}=1.607$. These are almost exact values for $K_{u}$ and $\omega_{u}$. However, if the slope is chosen
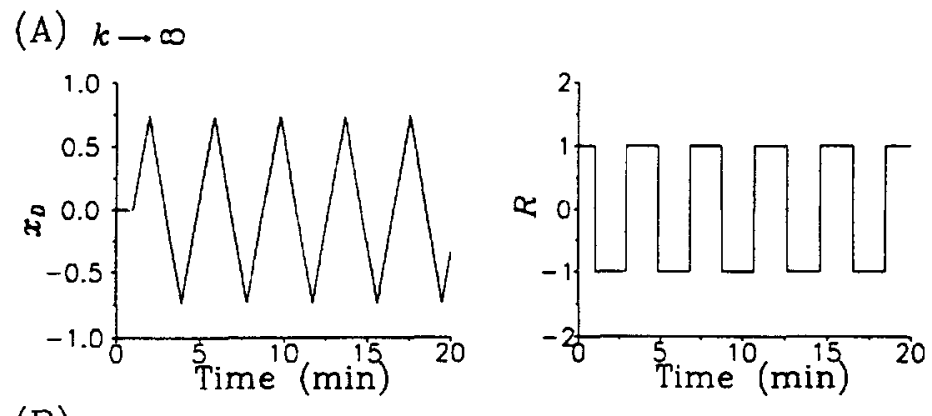

(B) $k=5$
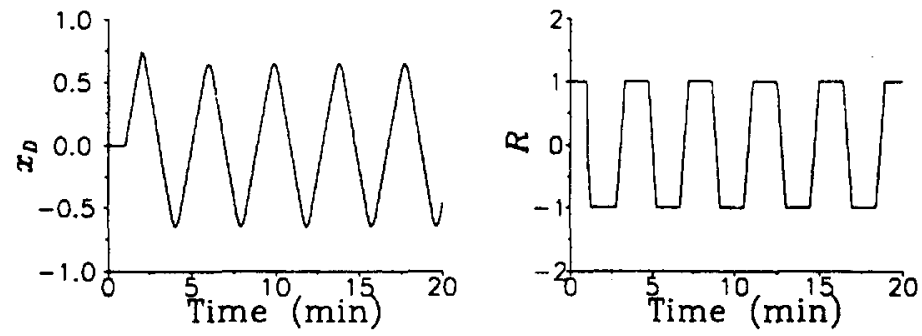

(C) $k=k_{\min }=2.1$
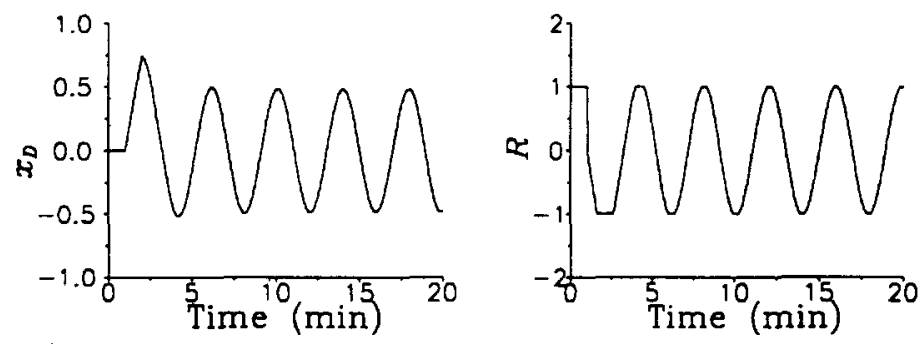

(D) $k=1.5$
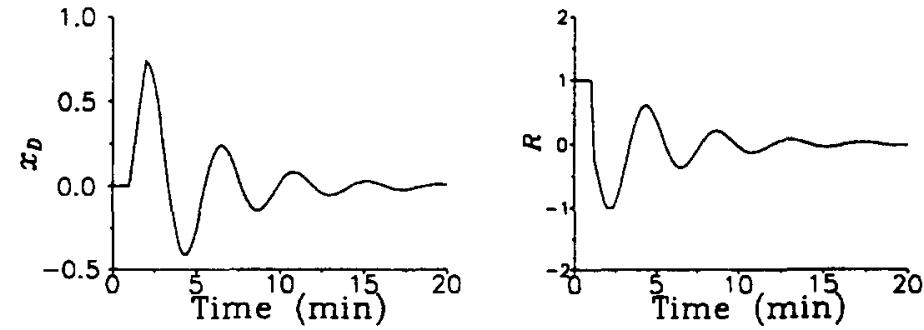

Fig. 7. Relay feedback test for Example 1: (A) ideal relay $(k \rightarrow \infty)$, (B) saturation relay $(k=5)$, (C) saturation relay $\left(k=k_{\min }=2.1\right)$, (D) saturation relay $\left(k=1.5<k_{\min }\right)$. 
to be less than $k_{\min }$ (e.g. $k=1.5$ ), then the relay fails to generate sustained oscillation.

The above example clearly indicates that the saturation relay can improve the estimation of ultimate gain and ultimate frequency. However, attention has to be paid in the selection of the slope $(k)$.

\section{AUTOTUNE IDENTIFICATION}

\subsection{Selection of the slope of saturation relay}

As mentioned earlier, a critical slope $\left(k_{\min }\right)$ exists to indicate the success/failure of a relay-feedback test. Furthermore, this critical slope is system dependent [eq. (29)]. Qualitatively, we also understand that the smaller the slope $(k)$ is, the more accurate estimates of $K_{u}$ and $\omega_{u}$ are, if the test is successful. However, in a relay-feedback test a quantitative value of the slope should be given.

In order to determine the slope, the typical process transfer function for chemical processes is used to illustrate the trade-off between success of an experiment and accuracy of the estimate. Consider a transfer function of the form

$$
G(s)=e^{-D s} /(\tau s+1)
$$

where $D$ is the dead time and $\tau$ is the time constant. A range of $D / \tau$ is studied for different value of dimensionless slope $\left(k / k_{\min }\right)$ and, subsequently, percent errors in $K_{u}$ and $\omega_{u}$ are evaluated. Results (Fig. 8) show that the improvement in the estimate levels off as $k$ approaches 10 times $k_{\min }$ (i.e. $k=10 k_{\min }$ ). Furthermore, the error in $K_{u}$ ranges from -10 to $-20 \%$ for these first-order plus dead time systems with an ideal relay $(k \rightarrow \infty)$ and the experiments tend to underestimate $K_{u}$. Several things become apparent immediately. First, generally, the slope should be less than $10 k_{\min }$ in order to improve the estimates. Second, it is preferable to choose the slope at least $1.4 k_{\min }$ to avoid unsuccessful relay-feedback test $\left(k_{\min }=\right.$ $\left.K_{u}=1 /\left|G\left(j \omega_{u}\right)\right|\right)$. Therefore, a simple value of thumb is to select the slope as 1.4 times $k_{\min }$. Notice that this is a safety factor for a class of transfer function over a range of parameter space; for a given system, the true safety factor is actually system dependent as shown in Fig. 8. In order to test the validity of this proposal, consider a second-order example.

\section{Example 2:}

$$
G(s)=37.7 e^{-10 s} /(7200 s+1)(2 s+1) .
$$

For this system, the exact values for $K_{u}$ and $\omega_{u}$ are 26.24 and 0.1315 . For an ideal relay $(k \rightarrow \infty)$, the ultimate gain found is 23.15 . This corresponds to $-11.7 \%$ error in $K_{u}$. As we decrease the slope to $K_{\min }$, almost exact value of $K_{u}$ can be found $\left(K_{u}=26.04\right)$ (Fig. 9). Furthermore, for $k=1.4 k_{\min }$, we can generate sustained oscillation with an improved estimate in both $K_{u}$ and $\omega_{u}$ (Fig. 9).
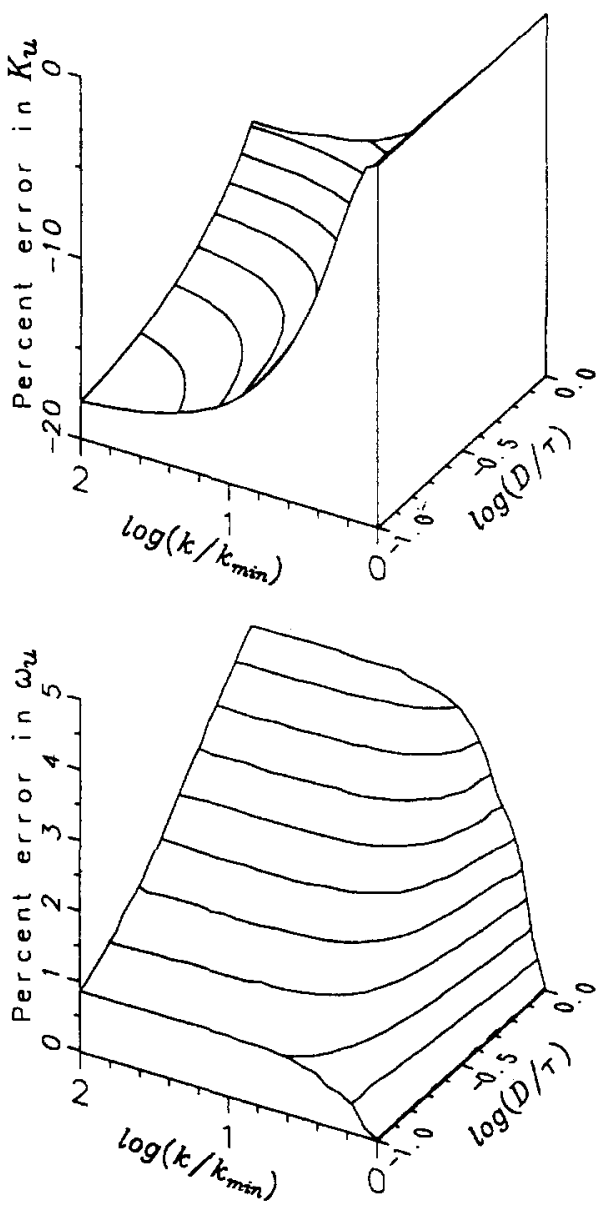

Fig. 8. Percent error in $K_{u}$ and $\omega_{u}$ for first-order plus dead time system $\left(e^{-D_{s}} /(\tau s+1)\right)$ with different value of $D / \tau$.

\section{Example 3:}

$$
G(s)=-(10 s+1) e^{-s} /(2 s+1)(4 s+1) .
$$

This is a system with inverse response (Shen and $\mathrm{Yu}$, 1994). The exact values for $K_{u}$ and $\omega_{u}$ are 0.576 and 0.336 , respectively. When an ideal relay is employed in the relay-feedback test, the percent errors in $K_{u}$ and $\omega_{u}$ are -15.8 and $-15.09 \%$, respectively. Again, improvement in the estimates of $K_{u}$ and $\omega_{u}$ can be seen as we decrease the slope toward $k_{\min }$ (Fig. 10). This example shows again that $k=1.4 k_{\min }$ will lead to quite accurate ultimate gain and ultimate frequency while guaranteeing the success of the relay-feedback test.

With this guideline in the selection of the slope of the saturation relay, we can devise a procedure for finding more accurate $K_{u}$ and $\omega_{u}$.

\subsection{Procedure}

Since $k_{\min }$ (or $K_{u}$ ) is needed to find the slope of the saturation relay, the proposed procedure finds a rough estimate of $k_{\min }$ first and goes on to find $k$ and 

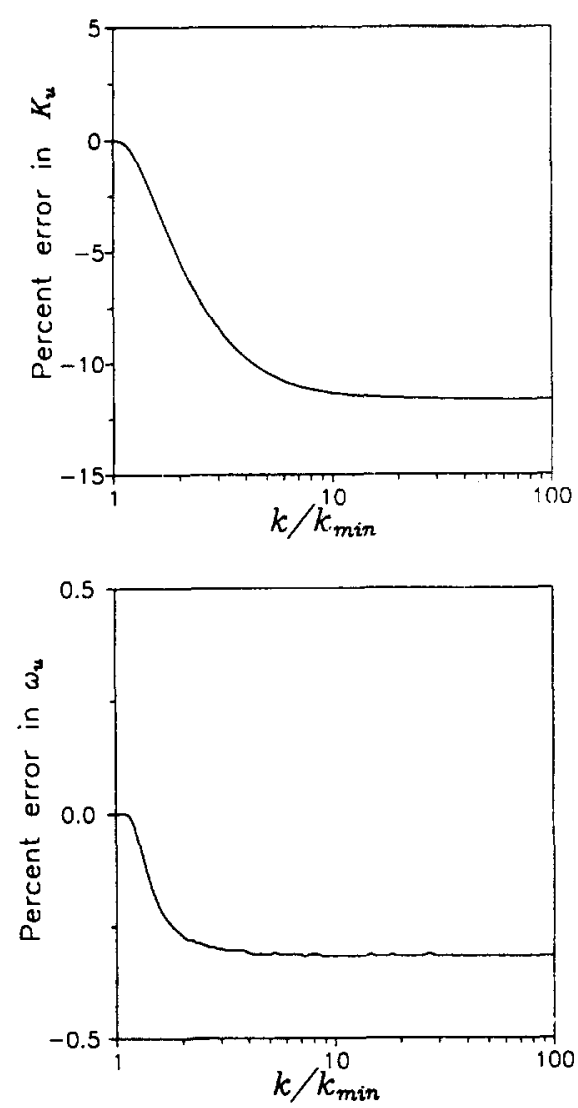

Fig. 9. Percent error in $K_{u}$ and $\omega_{u}$ for Example 2.

obtains a better $K_{u}$ and $\omega_{u}$. The procedure is summarized as follows. Consider a relay feedback system.

(1) Select the height of the relay $h$ (upper and lower limits in the manipulated input in the experiment).

(2) (a) Use ideal relay to estimate $\hat{K}_{u}\left(\hat{K}_{u}=4 h / \pi a\right)$.

(b) Calculate the slope of the saturation relay $k=1.4 k_{\min }\left(k_{\min }=\hat{K}_{u}\right)$.

(c) Continue the relay-feedback experiment using the saturation relay with $k=1.4 k_{\min }$.

(3) Find $K_{u}$ and $\omega_{u}$ from the relay feedback test.

\section{APPLICATIONS}

The proposed saturation relay feedback is applied to system identification (identifying $K_{u}$ and $\omega_{u}$ ) as well as autotuning multivariate systems. Both linear system and nonlinear process are studied.

Consider the WB column studied in Example 1. The exact value for $K_{u}$ and $\omega_{u}$ are 2.1 and 1.608 , respectively. The proposed procedure goes as follows. The relay height $h$ is chosen as unity. Initially, a positive change $R$ is made and $x_{D}$ starts to increase (Fig. 11). As soon as $x_{D}$ moves upward, $R$ is moved to the lower position $(\Delta R=-1)$. This ideal relay-feedback test goes on for $2-3$ cycles (e.g. time $<11 \mathrm{~min}$ in Fig. 11) and we can estimate $K_{u}$ from system responses. The result is $K_{u}=1.71$ ( $-18.6 \%$ error). With
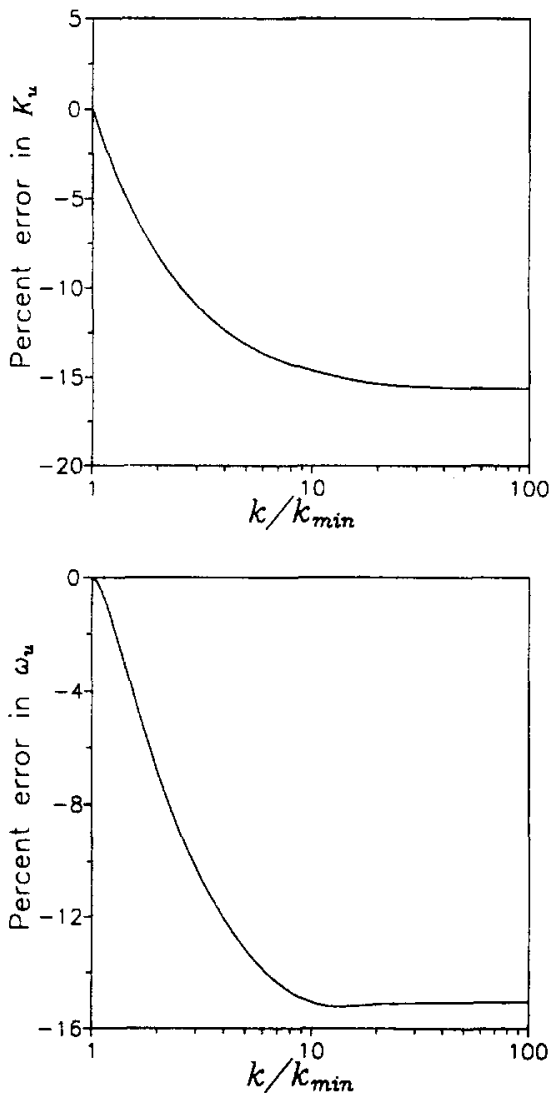

Fig. 10. Percent error in $K_{u}$ and $\omega_{u}$ for Example 3.

the initial result, the slope of the saturation relay is chosen as $k=1.4 k_{\min }=2.4$, then the relay feedback test continues with the saturation relay (e.g. time $>11 \mathrm{~min}$ in Fig. 11). The results show that the ultimate gain and ultimate frequency found from the saturation-relay feedback are 2.098 and 1.606 , respectively. This corresponds to $0.01 \%$ error in $K_{u}$ and $0.012 \%$ error in $\omega_{u}$. Obviously, significant improvement can be achieved using the proposed procedure.

A nonlinear distillation example is used to illustrate the accuracy of the proposed autotune identification procedure. The column studied by Shen and Yu (1992) is a 20-tray distillation column. The product specifications are 98 and $2 \%$ of the light component on the top and bottom of the column. The relative volatility is 2.26 with a reflux ratio 1.76 . Table 1 gives the steadystate values. The control objective is to maintain the top and bottom product compositions by changing the reflux flow rate $(R)$ and vapor boilup rate $(V)$. This is the conventional $R-V$ control structure (Fig. 12).

First, the $x_{B}-V$ loop is used to test the accuracy of proposed method in finding $K_{u}$ and $\omega_{u}$. Figure 13 shows the input $(V)$ and output $\left(x_{B}\right)$ responses using the proposed autotune identification with a relay height of $5 \%$. The results given in Table 2 , show that the ideal relay-feedback experiment gives significant errors in $K_{u}$ and $\omega_{u}$ compared with the stepping technique. On the other hand, saturation relay 

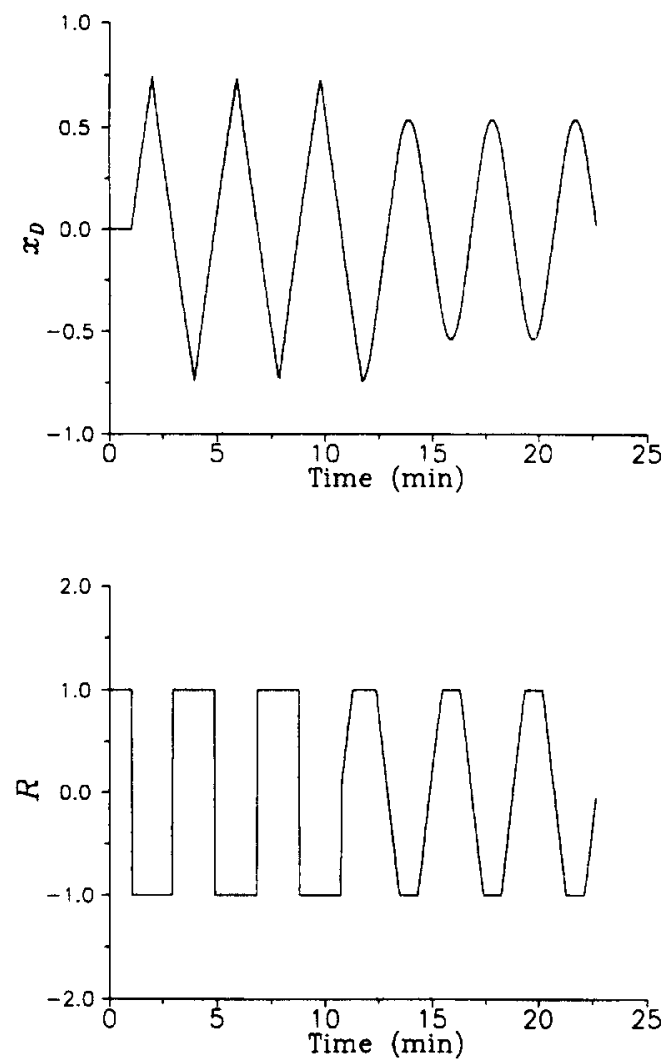

Fig. 11. Proposed autotune identification procedure for WB column.

Table 1. Steady-state values for moderate-purity distillation column

\begin{tabular}{lc}
\hline \multicolumn{1}{c}{ Parameters } & Values \\
\hline Number of trays & 20 \\
Feed tray & 10 \\
Relative volatility & 2.26 \\
Operating pressure (atm) & 1.0 \\
Feed flow rate $(\mathrm{kg} \mathrm{mole} / \mathrm{min})$ & 36.3 \\
Distillation flow rate $(\mathrm{kg} \mathrm{mole} / \mathrm{min})$ & 18.15 \\
Bottoms flow rate $(\mathrm{kg} \mathrm{mole} / \mathrm{min})$ & 18.15 \\
Reflux ratio & 1.76 \\
Feed composition (mole fraction) & 0.50 \\
Distillation composition (mole fraction) & 0.98 \\
Bottoms composition (mole fraction) & 0.02 \\
\hline
\end{tabular}

feedback with the slope $k=1.4 k_{\min }(k=704)$ gives very good estimate in $K_{u}$ and $\omega_{u}$. The errors in $K_{u}$ and $\omega_{\mu}$ are -2.8 and $-3.3 \%$, respectively.

Next, the saturation-relay feedback is applied to MIMO autotuning of this $R-V$ controlled column. The MIMO autotuning is performed sequentially starting from $x_{D}-R$ loop while keeping $x_{B}-V$ loop on manual. When $K_{u}$ and $\omega_{u}$ for the $x_{D}-R$ loop are found, the PI controller is tuned according to (Shen and $\mathrm{Yu}, 1994)$

$$
\begin{aligned}
K_{c} & =K_{u} / 3 \\
\tau_{I} & =P_{u} / 0.5 .
\end{aligned}
$$

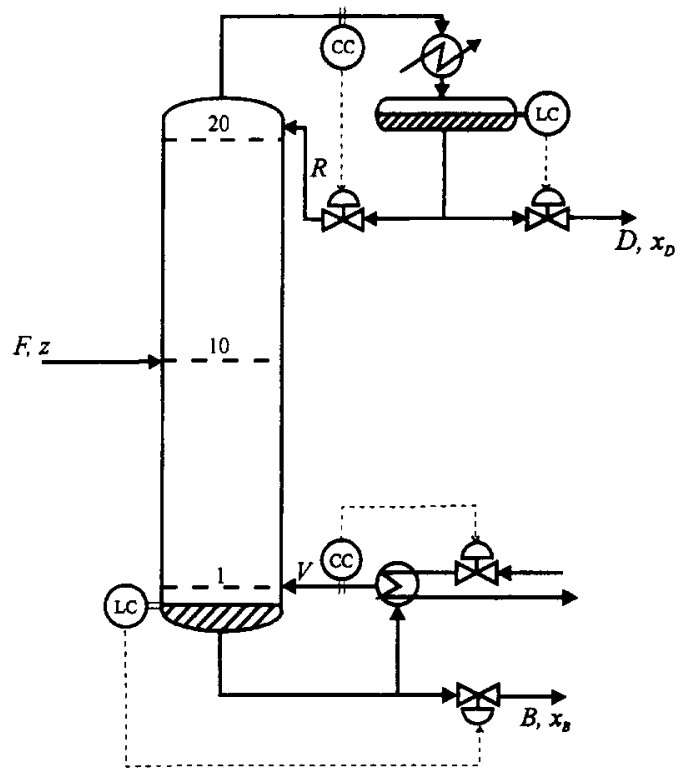

Fig. 12. $R-V$ controlled moderate-purity distillation column.

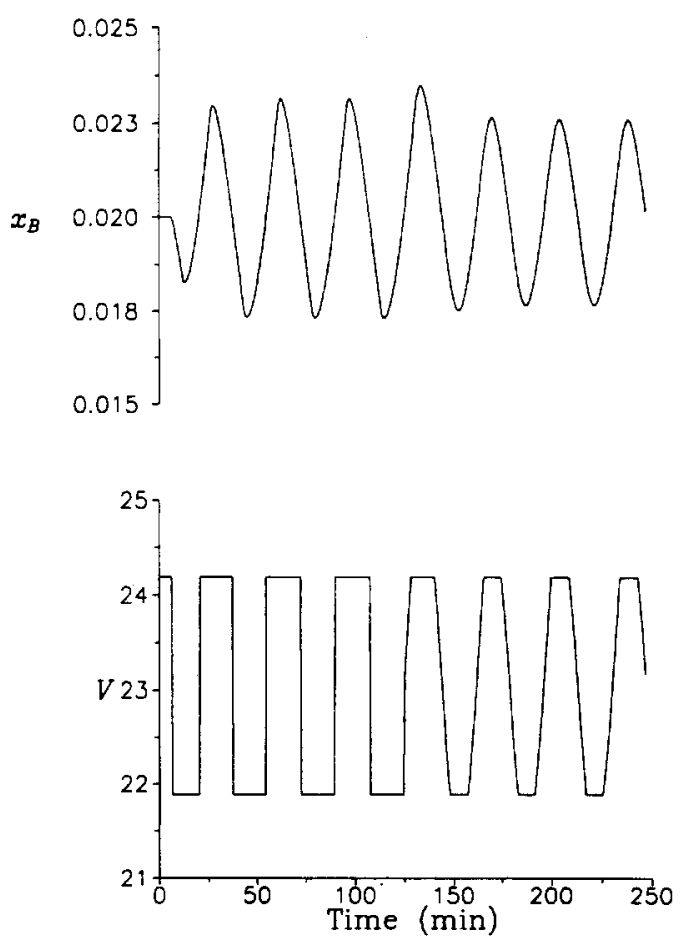

Fig. 13. Proposed autotune identification procedure in $x_{B}-V$ loop for moderate-purity distillation column.

With this set of tuning constants, the $x_{D}-R$ loop is closed and the saturation-relay feedback is performed on $x_{B}-V$ loop. This procedure is repeated until the tuning constants converge. Shen and $\mathrm{Yu}(1994)$ give details on this MIMO autotuning procedure. Figure 14 shows that, it takes 2 saturation-relay feedback test to complete the autotuning procedure. In order to 
Table 2. Identification results $\left(K_{u}\right.$ and $\left.\omega_{u}\right)$ in $x_{B}-V$ loop for moderate-purity distillation column

\begin{tabular}{lccccc}
\hline & \multicolumn{2}{c}{$K_{u}$} & & \multicolumn{2}{c}{$\omega_{u}$} \\
\cline { 2 - 3 } \cline { 5 - 6 } & Value & \% error & & Value & \% error \\
\hline Stepping method & 562.2 & 0 & & 0.1862 & 0 \\
Ideal relay $(k \rightarrow \infty)$ & 503.2 & -10.5 & & 0.1839 & -5.2 \\
Saturation relay $(k=704)$ & 547.1 & -2.8 & & 0.1802 & -3.3 \\
\hline
\end{tabular}

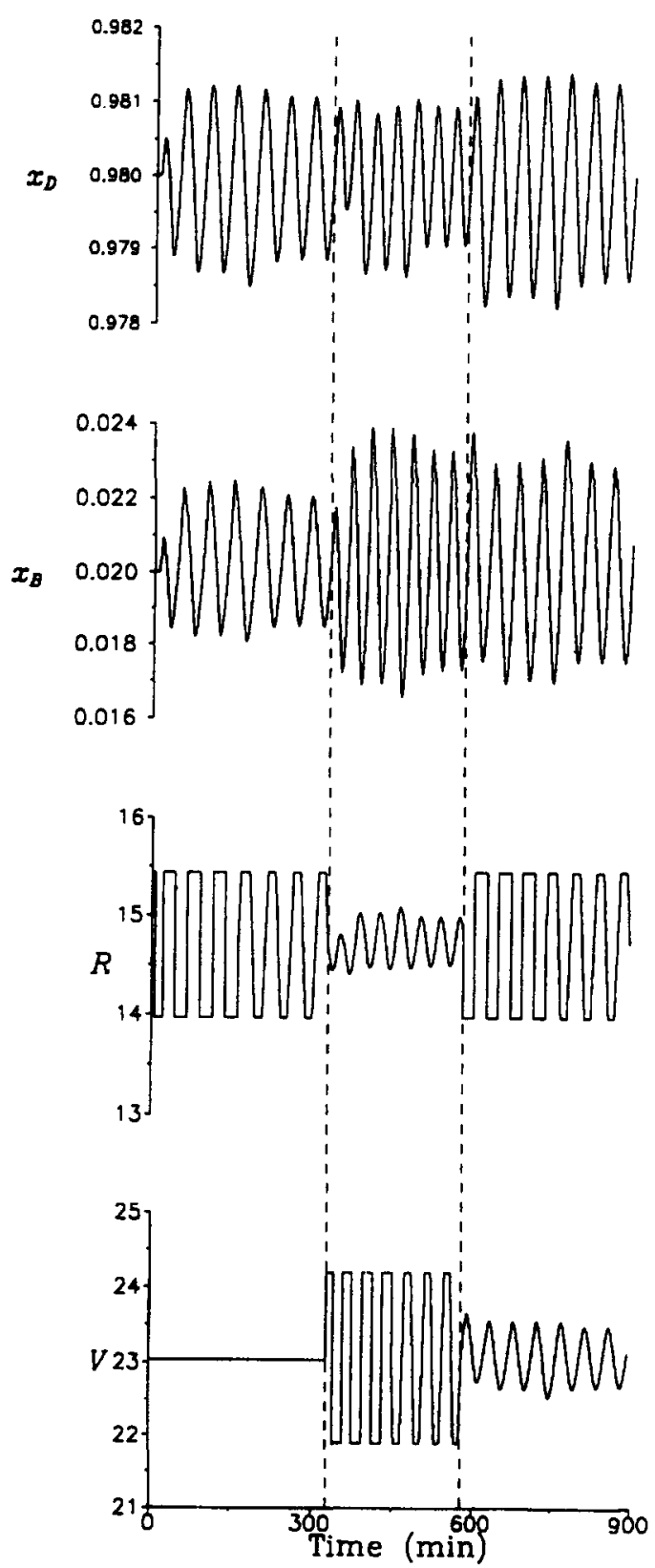

Fig. 14. MIMO autotuning process.

test the correctness of identified system, the closedloop transfer functions ( $g_{t i, C L}$ 's) obtained from different approaches are compared. Let 1 and 2 denote $x_{D}$ and $x_{B}$. The closed-loop transfer function for the
$x_{D^{-}}-R$ loop is

$$
g_{11, C L}=g_{11}\left(1-\frac{g_{12} g_{21}}{g_{11} g_{22}} \cdot \frac{g_{22} K_{2}}{1+g_{22} K_{2}}\right) \text {. }
$$

$K_{u}$ and $\omega_{u}$ from saturation-relay feedback (Fig. 14) are used to back-calculate the coefficients of the parametric $g_{11, C L}$ and $g_{22, C L}$.

$$
\begin{aligned}
& g_{11, C L}(s)=0.00965 e^{-6 s} /(9.89 s+1)(23 s+1) \\
& g_{22, C L}(s)=0.01316 e^{-6 s} /(4.7 s+1)(24.5 s+1) .
\end{aligned}
$$
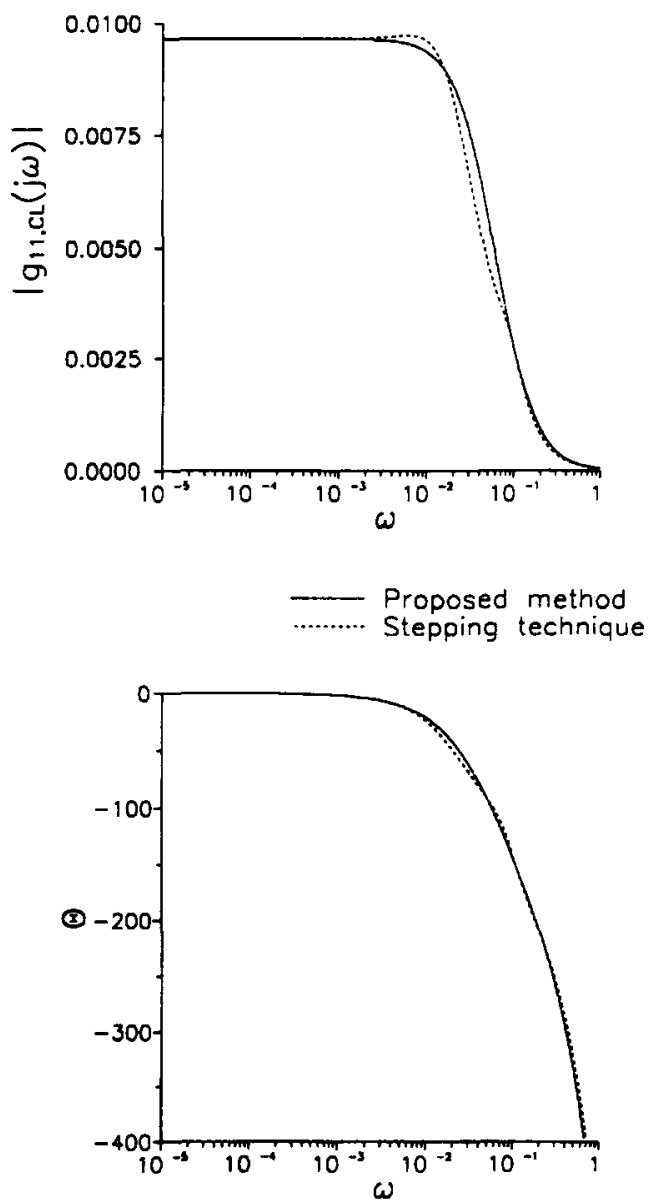

Fig. 15. Bode diagram for moderate-purity distillation column: (A) $g_{11, C L}$, (B) $g_{22, C L}$. 
(B)
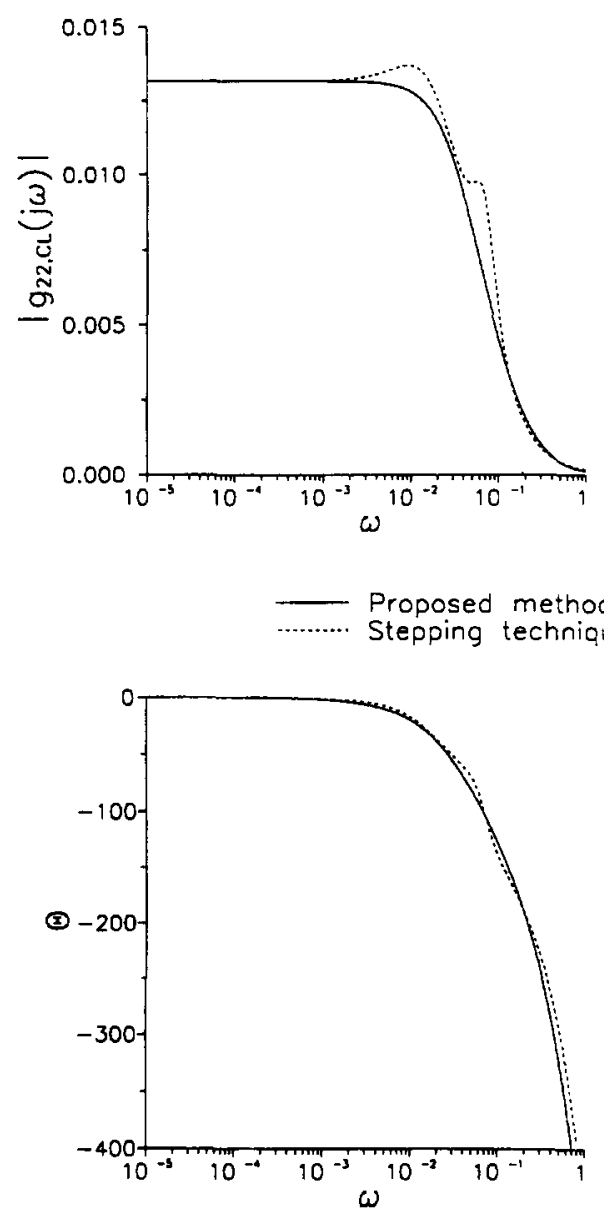

Fig. 15. (contd).

The two closed-loop transfer functions are compared with the frequency responses from the stepping technique. Results (Fig. 15) show that the proposed saturation-relay based MIMO autotuning gives very accurate estimates of the process transfer functions as compared to the almost analytical results (results from stepping technique). Furthermore, autotuning based on saturation-relay feedback gives satisfactory closedloop performance for $\pm 20 \%$ changes in feed composition (Fig. 16).

The linear and nonlinear examples as well as identification and autotuning results clearly indicate that the proposed saturation-relay feedback gives significant improvement in finding $\boldsymbol{K}_{\boldsymbol{u}}$ and $\omega_{\mathfrak{u}}$ and, subsequently, lead to improved performance in identification and MIMO autotuning.

\section{CONCLUSIONS}

In this work, the saturation-relay feedback system is proposed to improve the accuracy of the estimates

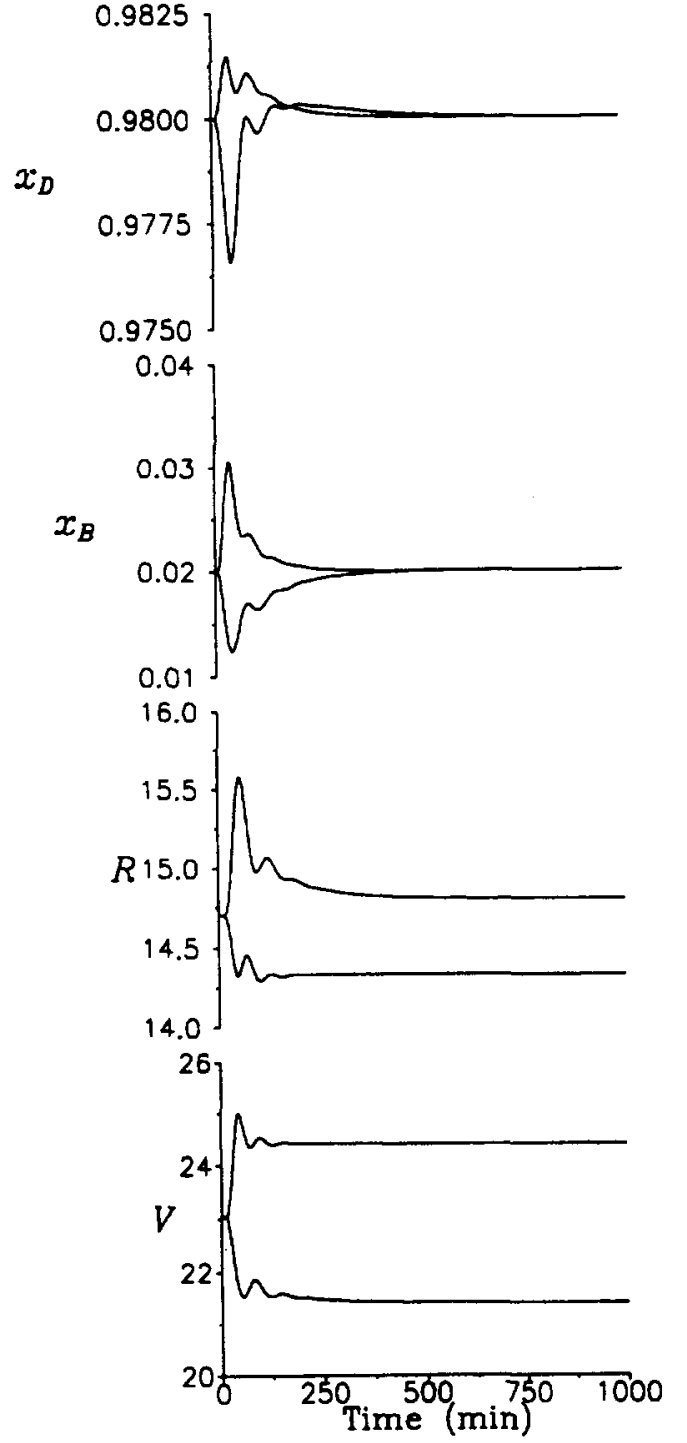

Fig. 16. Load responses for $\pm 20 \%$ feed composition changes.

of ultimate gain and ultimate frequency. The analyses show that significant improvement in the estimates of $K_{u}$ and $\omega_{u}$ can be achieved using saturation-relay feedback. It also shows that too small a slope in the saturation relay may fail to generate a limit cycle and, subsequently, lead to a failed experiment. A procedure to overcome the trade-off between the accuracy and (possible) failure is proposed. The proposed method is tested on linear and nonlinear systems. The results of system identification and MIMO autotuning show that significant improvement can be achieved. More importantly, the proposed method maintains the simplicity of the conventional relayfeedback system.

Acknowledgement-This work was supported by the National Science Council of the R.O.C. under the grant NSC 83-0402-E-011-057. 


$\begin{array}{ll}a & \text { amplitude of limit cycle } \\ D & \text { time delay } \\ e & \text { input to the nonlinear element } \\ \text { the }(i, j) \text { entry of process transfer function } & \text { matrix } \\ g_{i j}(s) & \text { the }(i, i) \text { entry of close-loop transfer func- } \\ g_{i i, C L}(s) & \text { tion } \\ G(s) & \text { process transfer function } \\ h & \text { magnitude of relay output } \\ k & \text { slope of saturation relay } \\ k_{\min } & \text { critical slope } \\ k_{p} & \text { steady-state gain } \\ K_{c} & \text { controller gain } \\ K_{i} & i \text { entry of multiloop SISO controller } \\ K_{u} & \text { ultimate gain } \\ \hat{R}_{u} & \text { estimated ultimate gain } \\ N(a) & \text { describing function } \\ P_{u} & \text { period of limit cycle } \\ R & \text { reflux flow rate } \\ s & \text { Laplace transform variable } \\ u & \text { output to the nonlinear element } \\ V & \text { vapor boilup flow rate } \\ x_{D} & \text { top component } \\ x_{B} & \text { bottoms component } \\ y & \text { process output } \\ & \end{array}$

\section{Greek letters}

$\begin{array}{ll}\alpha & \text { relative volatility } \\ \lambda_{i i} & (i, i) \text { entry of RGA } \\ \tau_{I} & \text { reset time of PI controller } \\ \omega & \text { frequency }\end{array}$

$\begin{array}{ll}\omega_{u} & \text { ultimate frequency } \\ \hat{\omega}_{u} & \text { estimated ultimate frequency }\end{array}$

\section{REFERENCES}

Atherton, D. P., 1982, Nonlinear Control Engineering. Van Nostrand Reinhold, New York.

Chiang, R. C., Shen, S. H. and Yu, C. C., 1992, Derivation of transfer function from relay feedback systems. Ind. Engng Chem. Res. 31, 855-860.

Chiang, R. C. and Yu, C. C., 1993, Monitoring procedure for intelligent control: on-line identification of maximum closed-loop log modules. Ind. Engng Chem. Res. 32, 90-99.

Hägglund, T. and Aström, K. J., 1991, Industrial adaptive controllers based on frequency response techniques. Automatica 27, 599-609.

Li, W., Eskinat, E. and Luyben, W. L., 1991, An improved autotune identification method, Ind. Engng Chem. Res. 30 , 1530-1541.

Lin, J. Y. and Yu, C. C., 1993, Automatic tuning and gain scheduling for Ph control. Chem. Engng Sci. 48, 3159-3171.

Loh, A. P. and Vasnani, V. U., 1994, Describing function matrix for multivariable systems and its use in multiloop PI design. J. Proc. Control 4, 115-120.

Luyben, W. L., 1987, Derivation of transfer functions for highly nonlinear distillation columns. Ind. Engng Chem. Res. 26, 2490-2495.

Luyben, W. L. and Eskinat, W., 1994, Non-linear autotune identification. Int. J. Control 59, 595-626.

Papastathopoulou, H. S. and Luyben, W. L., 1990, Tuning controllers on distillation columns with the distillate-bottoms structure. Ind. Engng Chem. Res. 29, 1859-1868.

Shen, S. H. and Yu, C. C., 1992, Indirect feedforward control: multivariable systems. Chem. Engng Sci. 47, 3085-3097.

Shen, S. H. and Yu, C. C., 1994, Use of relay-feedback test for automatic tuning of multivariable systems. A.I.Ch.E. J. 40, 627-646.

Wood, R. K. and Berry, M. W., 1973, Terminal composition control of a binary distillation column. Chem. Engng Sci. 28, 707-720. 